\title{
RELEITURA DA PRIVACIDADE DIANTE DAS NOVAS TECNOLOGIAS: REALIDADE AUMENTADA, RECONHECIMENTO FACIAL E INTERNET DAS COISAS
}

\author{
Guilherme Misugi* \\ Cinthia Obladen de Almendra Freitas** \\ Antônio Carlos Efing ****
}

SUMÁRIO: Introdução; 2 A Proteção do Cidadão-consumidor na Sociedade de Informação; 3 A Inovação Tecnológica e o Risco à Privacidade; 3.1 Realidade Aumentada e o Reconbecimento Facial; 3.2 A Internet das Coisas, Dispositivos Móveis e seus Aplicativos e Tecnologia "Wearable"; 4 Releitura da Privacidade; 5 Considerações Finais; Referências.

RESUMO: $\mathrm{O}$ artigo apresenta uma releitura da privacidade não se limitando à sua conceituação jurídica positivada, mas considerando o contexto sociocultural no qual se desenvolve, bem como sua dinamicidade diante das novas tecnologias. Analisase a proteção do cidadão-consumidor na sociedade da informação, o modo como as novas tecnologias têm alterado a relação interpessoal e a interação com o meio, bem como busca uma releitura da privacidade a partir do contexto sociocultural em que se insere este indivíduo, cidadão e consumidor. A partir de revisão bibliográfica e método dedutivo, o artigo fornece uma interpretação de algumas entre as muitas novas tecnologias disponíveis na sociedade contemporânea, exemplificando as revoluções tecnológicas que têm o potencial de alterar o modo como se interpreta a privacidade. Foram elencadas as seguintes tecnologias: realidade aumentada, reconhecimento facial e as particularidades dos dispositivos móveis e Internet das coisas. Por fim, reconhece-se que uma concepção estática de privacidade não se demonstra suficiente para sua efetividade, sendo necessária uma releitura dinâmica, contextualizada e principiológica que assegure o direito à privacidade nas relações públicas e privadas do cidadão-consumidor imerso em tecnologias cada vez mais acessíveis e transparentes.

PALAVRAS-CHAVE: Novas tecnologias; Privacidade; Proteção do cidadãoconsumidor; Sociedade da informação.

\footnotetext{
Mestre no Programa de Pós-graduação em Direito Socioambiental e Sustentabilidade pela Pontifícia Universidade Católica do Paraná (PUCPR); Bolsista CAPES; Advogado; Curitiba (PR), Brasil. E-mail: guilhermemisugi@gmail.com

** Doutora em Informática pela Pontifícia Universidade Católica do Paraná (PUCPR); Docente Titular Permanente do Programa de Pós-Graduação em Direito (PUCPR), Curitiba (PR), Brasil.

${ }^{* * *}$ Doutor e Docente titular do Programa de Pós-graduação Lato sensu e Stricto sensu da Pontifícia Universidade Católica do Paraná (PUCPR); Docente de Graduação; Membro do Instituto dos Advogados do Paraná; Advogado em Curitiba (PR), Brasil.
} 


\title{
RE-INTERPRETATION OF PRIVACY WITHIN THE CONTEXT OF NEW TECHNOLOGIES: INCREASED REALITY, FACIAL RECOGNITION AND INTERNET
}

\begin{abstract}
Current paper provides a new interpretation of privacy without limiting its positive juridical concept, taking into consideration the socio-cultural context in which it develops and its dynamics in the wake of new technologies. The protection of the citizen-consumer in the society of information is analyzed, coupled to the manner the new technologies have changed inter-personal relationships and interactivity with the environment. A re-interpretation of privacy is given from the socio-cultural context in which the person, as citizen and consumer, is inserted. Through a bibliographical review and the deductive method provided, the paper interprets some of the new technologies available in modern society, with examples of technological revolutions capable of changing the manner in which privacy is interpreted. Increased reality, facial recognition and the details of movable disposition and Internet are the technologies discussed. A static concept of privacy is not sufficient for its efficaciousness. A dynamic interpretation, contextualized and based on principles, is required that would guarantee the right to privacy in the public and private relationships of the citizen-consumer immersed in greatly accessible and transparent technologies.
\end{abstract}

KEY WORDS: Information society; Privacy; New technologies; Protection of the citizen-consumer.

\section{RELECTURA DE LA PRIVACIDAD FRENTE A LAS NUEVAS TECNOLOGÍAS: REALIDAD AUMENTADA, RECONOCIMIENTO FACIAL E INTERNET DE LAS COSAS}

RESUMEN: El artículo presenta una relectura de la privacidad no limitándose a su concepto jurídico positivado, pero considerando el contexto sociocultural en el cual se desarrolla, bien como su dinámica frente a las nuevas tecnologías. Se analiza la protección del ciudadano-consumidor en la sociedad de información, el modo cómo las nuevas tecnologías alteran la relación interpersonal y la interacción con el medio, bien como busca una relectura de la privacidad a partir del contexto sociocultural en que está inserido el individuo, ciudadano y consumidor. A partir de la revisión bibliográfica y método deductivo, el artículo presenta una interpretación de algunas entre las muchas tecnologías disponibles en la sociedad contemporánea, ejemplificando las revoluciones tecnológicas que poseen potencial de alterar el modo como se interpreta la privacidad. Fueron enseñadas las siguientes tecnologías: realidad aumentada, reconocimiento facial y las particularidades de los dispositivos 
móviles e Internet de las cosas. Por fin, se reconoce que una concepción estática de la privacidad no se demuestra suficiente para su efectividad, siendo necesaria una relectura dinámica, contextualizada y principio-lógica que asegure el derecho a la privacidad en las relaciones públicas y privadas del ciudadano-consumidor inmerso en tecnologías cada vez más accesibles y transparentes.

PALABRAS-CLAVE: Nuevas tecnologías; Privacidad; Protección del ciudadanoconsumidor; Sociedad de información.

\section{INTRODUÇÃO}

Ao se proclamar atualmente a afirmação de que a mera comercialização de máquinas fotográficas representaria um risco à privacidade dos cidadãos e prejudicaria até mesmo a santidade do lar, obviamente esta seria, imediata e coerentemente, refutada. Ocorre que no final do século XIX, a outrora inovação tecnológica e sua comercialização eram acompanhadas de diversas críticas e riscos não compreendidos que impulsionaram publicações jurídicas, que se demonstram relevantes até os dias atuais. O trabalho de Warren e Brandeis ${ }^{1}$ pontua em 1890, época em que nem se falava em computadores, celulares e Internet, que os indivíduos têm e sempre terão total proteção sobre a pessoa e a propriedade, sendo portanto o conceito de privacidade muito antigo. Porém, necessita-se de tempos em tempos rever a natureza e a extensão de tal proteção, visto que a discussão dos autores é pertinente à privacidade almejada pela sociedade tecnológica atual.

$\mathrm{O}$ artigo contextualiza esta questão entre a privacidade e as novas tecnologias à sociedade da informação, na qual a revolução tecnológica tem se desdobrado constantemente em novas e, por vezes, intrusivas possibilidades.

Não se pretendendo esgotar o tema ou mesmo conceituar precisamente a tutela jurídica do direito à privacidade, busca-se a construção sociocultural da noção de privacidade e uma dinâmica releitura para que permaneça eficaz diante destas novas tecnologias. Foram apresentadas algumas entre as muitas tecnologias existentes, destacando-se: realidade aumentada, reconhecimento facial e as particularidades dos dispositivos móveis e Internet das coisas. Não é objetivo tratar das características técnicas de tais tecnologias, mas sim do modo como tais

${ }^{1}$ WARREN, Samuel D.; BRANDEIS, Louis D. The Right to Privacy. Harvard Law Review, v. 4, n. 5, 1890. Disponível em: < http://faculty.uml.edu/sgallagher/Brandeisprivacy.htm> Acesso em: 30 jul. 2015. p. 01. 
tecnologias estão se tornando cada vez mais presentes no dia a dia das pessoas e influenciando o modo de vida do cidadão-consumidor.

A informação está acessível a partir de qualquer lugar e vive-se um cenário no qual não se está atento a tudo isso e todo o tempo. Vive-se a realidade prevista por Weiser ${ }^{2}$, sob a ótica da computação ubíqua, possibilitada pelo desenvolvimento da computação móvel, com a aplicação de sistemas embarcados, e pela computação pervasiva, a qual é entendida pela capacidade dos meios de computação estar distribuídos, de forma imperceptível, nos diferentes ambientes em que se encontram os usuários ${ }^{3}$.

Considerando-se este contexto, inicia-se o presente estudo esboçando as principais características da sociedade de consumo, caracterizada por ser a sociedade da informação, e a permanente necessidade de atualização do Direito como ciência para a manutenção dos valores definidos como essenciais à sociedade.

Posteriormente, são apresentadas as novas tecnologias e os riscos que representam à privacidade, visto que são caracterizados por uma capacidade de processamento e armazenagem nunca antes imaginados e que, principalmente, têm a capacidade de modificar significativamente o modo como as pessoas interagem com o viver em sociedade e com as demais pessoas.

Por fim, realiza-se uma releitura da privacidade, partindo de uma breve introdução jurídica e aprofundando-se na construção sociocultural deste valor, apontando-se assim que sua dinamicidade e amplitude, por vezes nebulosas, dificultam uma interpretação jurídica adequada, uma vez que é igualmente complexa.

$\mathrm{O}$ artigo segue método dedutivo de pesquisa para relacionar o conceito de privacidade às novas tecnologias, discutindo o entendimento e os limites frente ao Código de Defesa do Consumidor e ao Marco Civil da Internet. Desse modo, questiona-se: A proteção do cidadão-consumidor frente às novas tecnologias é adequada e eficiente? É a privacidade um conceito ainda em construção? Como o Direito, a partir de uma construção sociocultural, compreende a privacidade frente às novas tecnologias? Muitos são os questionamentos e, portanto, tais aspectos são merecedores de atenção e estudo.

\footnotetext{
${ }^{2}$ WEISER, Mark. Some computer science issues in ubiquitous computing. Communications of the ACM. Disponível em: < http://www.ubiq.com/hypertext/weiser/UbiCACM.html>. Acesso em: 20 out. 2014.

${ }^{3}$ MOUTINHO, Ana Maria. Inteligência ambiente: contributo para a conceptualização de parede inteligente. 2010. Dissertação (Mestrado) - Universidade de Lisboa, Lisboa, 2010. Disponível em: < http://repositorio. ul.pt/bitstream/10451/7277/2/ULFBA_tes\%20392.pdf> Acesso em: 30 jul. 2015.
} 


\section{A PROTEÇÃO DO CIDADÃO-CONSUMIDOR NA SOCIEDADE DE INFORMAÇÃO}

A revolução informacional provocada pela utilização dos computadores e da Internet alterou o modo e ritmo de processamento de informações, refletindo consequentemente na redefinição da própria sociedade de consumo. Representando, em realidade, significativas contribuições convergentes a uma sociedade de consumo caracterizada precisamente por esta urgência e globalização.

Os três principais fenômenos que marcaram a revolução informacional são apontados por Barreto Junior como convergência da base tecnológica, dinâmica da indústria e o crescimento e expansão da Internet. Segundo o qual, ainda, "definese a Sociedade da Informação como uma nova era, na qual a informação adquire centralidade e passa a fluir a velocidade e em quantidades ante inimagináveis, assumindo valores sociais e econômicos fundamentais"

Trata-se, na concepção de alguns autores, como Benjamin'5 , Gibran e Efing, da sociedade de informação como um desdobramento da sociedade de consumo, definida pela "diminuição da distância e do tempo (como outrora concebido), a diluição de barreiras (a globalização), pelo excesso de dados e de informações, pela ubiquidade, dentre outras características" ${ }^{\circ}$. E complementando esta conceituação, Lisboa apresenta que:

A Sociedade da Informação, como se pôde verificar, é a sociedade posterior à Revolução causada pela introdução do computador nas relações jurídicas. [...] Trata-se de um ambiente de atuação da pessoa, inclusive na órbita negocial, que aperfeiçoou os sistemas de bens de produção e de comunicação, a partir da invenção do computador?

Ressalva-se que nesta sociedade de informação, o convívio social e relações jurídicas foram amplamente alterados, reforçando a necessidade da revisão do direito que as baliza, revisitando-o em todas suas áreas à luz da sociedade de informação: 0 direito civil, o direito empresarial, o direito do consumidor, o direito processual, o direito do trabalho, o direito tributário, o direito administrativo, entre outros ${ }^{8}$.

${ }_{4}^{4}$ BARRETO JUNIOR, Irineu Francisco. Abordagens recentes da pesquisa jurídica na Sociedade da Informação. In: PAESANI, Liliana Minardi. (Coord.). O direito na sociedade de informação II. São Paulo: Atlas, 2009, p. 40.

5 BENJAMIN, Antônio Herman et al. Código brasileiro de defesa do consumidor: comentado pelos autores do Anteprojeto. 9. ed. Rio de Janeiro: Forense Universitária, 2007, p. 416.

${ }^{6}$ GIBRAN, Fernanda Mara; EFING, Antônio Carlos. Banco de Dados de Consumo como instrumento para o desenvolvimento da sociedade de informação. In: ENCONTRO NACIONAL DO CONPEDI, 19., Fortaleza. Anais... Fortaleza: CONPEDI, 2010. p. 3886-3895, p. 3887.

7 LISBOA, Roberto Senise. O consumidor na sociedade da informação. Revista de Direito do Consumidor, n. 61, jan. 2007, p. 214.

8 Idem. 
Concorda-se, portanto, com o apontado por Miragem que defende que "a discussão sobre os limites de aplicação das normas jurídicas às relações estabelecidas por intermédio da Internet diz respeito, em verdade, à eficácia e efetividade da norma na regulação de um determinado suporte fático sobre o qual deve incidir" ".

Neste sentido, mesmo que o cerne do presente estudo e a releitura da privacidade diante das novas tecnologias não se concentrem nas questões unicamente consumeristas, envolvendo os direitos da personalidade de todos os cidadãos, não se pode desconsiderar o papel nuclear que o consumo assumiu na sociedade atual, bem como a tutela jurídica envolvendo os arquivos de consumo igualmente essenciais para a abordagem do tema.

Especificamente, acerca das práticas mercadológicas, essa se insere na tutela conferida pelo CDC em seu capítulo V que versa sobre as práticas comerciais, e é caracterizada por uma proteção ampla, mas abstrata (art. 29 da Lei 8.078/90). Esta análise se torna relevante ao presente estudo, uma vez que tanto os arquivos de consumo como as demais práticas mercadológicas que passarão a ser analisadas no capítulo seguinte estão sob esta tutela principiológica conferida pelo Código de Defesa do Consumidor.

Complementam os autores do anteprojeto do CDC que tendo em vista esta busca de um mercado de consumo e ordem econômica convergente com os valores socioeconômicos expostos, deve-se buscar a harmonização dos agentes econômicos e as práticas comerciais, não com o intuito de reprimi-la, mas apenas para colocá-la a serviço do interesse público ${ }^{10}$.

Trata-se, portanto, de uma interpretação jurídica que aproxima as novas tecnologias a um solidarismo entre os agentes econômicos, partindo de uma cooperação "assentada no princípio da dignidade pessoal, do respeito, que se presta como conduta que não apenas o outro espera do seu parceiro negocial (expectativa), mas também reflexamente a coletividade, num sentido de comprometimento pela segurança do tráfico mediante a adoção de contratos justos"11.

Em outro momento, Misugi e Efing (2014) defenderam precisamente que esta atuação mercadológica solidária de todos seus agentes, com respeito

9 MIRAGEM, Bruno. Responsabilidade por danos na sociedade de informação e proteção do consumidor: desafios atuais da regulação jurídica da Internet. Revista de Direito do Consumidor, São Paulo, n. 70, p. 41-92, abr. 2009. p. 44.

${ }^{10}$ BENJAMIN, Antonio Herman V. et al. Código Brasileiro de defesa do consumidor: comentado pelos autores do anteprojeto. 10. ed. revista, atualizada e reformulada. Rio de Janeiro: Forense, 2011, Vol. I. p. 260.

${ }^{11}$ LISBOA, Roberto Senise. Tecnologia, confiança e sociedade: por um novo solidarismo. In: PAESANI, Liliana Minardi. (Coord.). O direito na sociedade de informação II. São Paulo: Atlas, 2009, p. 65. 
aos direitos fundamentais do cidadão consumidor trata-se de pressuposto para o desenvolvimento socioambiental, mormente diante da revolução informacional que se apresentou.

$\mathrm{Na}$ urgência das novas liberdades possibilitadas pelas tecnologias que se inserem as novas complexidades envolvendo este forte desejo de comunicação imediatista e o direito à privacidade, devendo este ser atualizado e recomposto de acordo com as características desta sociedade da informação altamente dinâmica.

\section{A INOVAÇÃo TECNOLÓGICA E O RISCO À PRIVACIDADE}

Cada um dos instrumentos revolucionários descritos no início da Sessão 1 proveio de uma evolução tecnológica que ao mesmo tempo facilitou o desenvolvimento de alguma atividade específica e desencadeou diversas consequências reflexas outrora inimagináveis, efetivamente alterando a relação do homem com o meio.

Esta imprevisibilidade representa uma das características da inovação tecnológica, visto que somente se compreenderão todos seus efeitos diretos e reflexos após a sua contextualização sociocultural.

Para exemplificar este raciocínio remete-se à obra paradigmática de Warren e Brandeis, The right to privacy, de 1890, na qual se previu que os recintos sagrados da vida privada e doméstica haviam sido invadidos pelas fotografias instantâneas e empreendimentos jornalísticos, de modo que o que antes era sussurrado na intimidade de um closet passaria a ser proclamado nas coberturas das casas ${ }^{12}$.

Mais de um século se passou e pôde se constatar que as vantagens provenientes desta inovação tecnológica sobrepuseram os eventuais infortúnios envolvendo a vida privada dos cidadãos, existindo, em realidade, uma adequação e resiliência cultural que harmonizaram os valores existentes.

Mesmo assim estas preocupações têm acompanhado todas as grandes inovações tecnológicas, de modo que os riscos apontados pelos autores norteamericanos permanecem atuais com a sociedade de informação, podendo ser novamente contextualizadas com as, cada vez mais frequentes, inovações tecnoinformacionais.

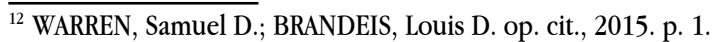


De modo que se questiona efetivamente se a vida privada e íntima permanece assegurada, mesmo com tantas inovações como, não mais a câmera fotográfica instantânea, mas a propagação da realidade aumentada e reconhecimento facial, a popularização dos dispositivos móveis, cada vez mais integrantes ao cotidiano e ao corpo humano, e seus aplicativos.

\subsection{REALIDADE AUMENTADA E O RECONHECIMENTO FACIAL}

A noção básica da realidade aumentada possui certa similaridade com as tecnologias óticas que utilizavam de instrumentos mecânicos, como os binóculos por exemplo, para se visualizar o mundo sob outra perspectiva. E nos últimos anos tem se utilizado da capacidade de processamento de imagens e dados dos dispositivos móveis com este mesmo intuito, integrar uma realidade virtual a um contexto físico existente.

Ao se compreender esta possibilidade de interação imediata e abastecida pelas informações da Internet, criou-se a expectativa de uma alteração paradigmática que era concebida até mesmo como o desenvolvimento de um sexto sentido, como exemplifica a organização AR.ORG que trata do tema:

In the early days, augmented reality promised to change the way we interact with the world in every single field, a force to accelerate progress; a technology that would digitize interaction with the real world and would enable people to master skills effortlessly, increase situational awareness with a 'digital sixth sense', and have more fun-away from the screen. Augmented reality meant augmented bumanity ${ }^{13}$.

De acordo com esta mesma entidade, estima-se que até 2020 mais de um bilhão de pessoas terão utilizado ativamente esta tecnologia da realidade aumentada, tendo implicações em diversas áreas como o entretenimento, design de produtos, publicidade, marketing, entre outros.

${ }^{13}$ Tradução livre: "Nos primeiros dias, realidade aumentada prometeu mudar a forma como interagimos com o mundo em cada área isolada, uma força para acelerar o progresso; uma tecnologia que digitalizaria a interação com o mundo real e permitiria que as pessoas a dominassem a habilidades sem esforço, aumentando a consciência situacional com um 'sexto sentido digital', e se divertir mais - longe da tela. A realidade aumentada significava humanidade aumentada". Disponível em: <http://www.augmentedreality.org/\#!our-story/c1lhp>. Acesso em: 30 jul. 2015. 
Para ilustrar o potencial de desenvolvimento desta realidade aumentada, destaca-se sua convergência com outras tecnologias desenvolvidas recentemente, como o reconhecimento facial, que tem a capacidade de alterar significativamente diversas atividades cotidianas.

De acordo com Welinder, a tecnologia de reconhecimento facial visa combinar as desenvolvidas percepções humanas com a imensa capacidade de processamento e armazenamento dos computadores ${ }^{14}$. E cuja definição, de acordo com Lavashov, pode ser assim descrita:

Facial recognition technology uses a photographic camera combined with facial recognition software. This software is able to detect and isolate buman faces captured by the camera and analyze them using an algorithm that extracts identifying features. The algorithm identifies and measures "nodal points" on the face, which are defined by the peaks and valleys that make up human facial features. Using these measurements, the algorithm determines an individual's identifying characteristics, such as distance between the eyes, width of the nose, shape of cheekbones, and the length of the jawline ${ }^{15}$.

Não se trata de tecnologia estritamente inédita visto que tem sido desenvolvida há mais de quarenta anos, mas que teve recentes progressos consideráveis com a denominada evolução da web 2.0 ${ }^{16}$. Para exemplificar esta evolução, Acquisti et al. (2014, p. 01) descrevem que o programa utilizado pelo Departamento de Defesa do Governo Norte-Americano apresentava em 1997: "an error rate of 0.54 (the false reject rate at a false accept rate of 1 in 1,000). By 2006, the best recognizer scored 0.026. By 2010, the best recognizer scored 0.003 an improvement of more than two orders of magnitude in just over 10 years" 17 .

Welinder exemplifica esta evolução tecnológica ao descrever que na etapa

\footnotetext{
${ }^{14}$ WELINDER, Yana. A Face Tells More than a Thousand Posts: Developing Face Recognition Privacy in Social Networks (July 16, 2012). Harvard Journal of Law and Technology, v. 26, n. 1, 2012, p. 170.

15 Tradução livre: "A tecnologia de reconhecimento facial usa uma câmera fotográfica combinado com um software de reconhecimento facial. Este software é capaz de detectar e isolar rostos humanos capturados pela câmera e analisá-los usando um algoritmo que extrai e identifica características. $\mathrm{O}$ algoritmo identifica e mede 'pontos nodais' na face, que são definidos pelos picos e vales que compõem as características faciais humanas. Utilizando estas medições, o algoritmo determina as características de identificação de um indivíduo, tais como a distância entre os olhos, largura do nariz, de forma maçãs do rosto, e o comprimento da linha da mandíbula". LEVASHOV, Kirill. The Rise of a New Type of Surveillance for Which the Law Wasn't Ready (2013). Columbia Science and Technology Law Review, v. 15, p. 164, Fall 2013. p. 167-168.

${ }^{16}$ ACQUISTI, Alessandro; GROSS, Ralph; STUTZMAN, Fred. Face Recognition and Privacy in the Age of Augmented Reality. Journal of Privacy and Confidentiality, v. 6, n. 2, Article 1. 2014, p. 1.

${ }^{17}$ Tradução livre: "Uma taxa de erro de 0,54 (a taxa de falso rejeitar a uma taxa aceitar falso de 1 em 1000). Em 2006, o melhor reconhecedor marcou 0,026. Até 2010, o melhor reconhecedor marcou 0.003 , uma melhoria de mais de duas ordens de magnitude em pouco mais de 10 anos". WELINDER, Yana, op. cit., 2012, p. 171.
} 
inicial de desenvolvimento era possível apenas a identificação individual de uma face a partir de uma visão frontal e com a iluminação uniforme, de modo que se comprovou que recentemente podem ser identificados vários rostos simultaneamente, sob vários ângulos e em meio a um complexo plano de fundo. $\mathrm{E}$, conforme pesquisa realizada na Carnegie Mellon University, a partir desta tecnologia e utilização de dados disponíveis no Facebook foi possível a identificação de 31,18\% dos presentes em seu campus. Vale destacar que, com esta tecnologia, a partir da simples presença do estudante no local monitorado foi possível o acesso a diversos dados pessoais relevantes ${ }^{18}$.

O potencial transformador desta tecnologia já chamou a atenção das grandes empresas tecnológicas, de modo que com a aquisição da Neven Vision, Like.com e Pittpatt, o próprio Google tem investido no reconhecimento facial; a Apple também implementou este serviço à Apple's iPboto a partir de 2009; sendo igualmente utilizado pelo Facebook na denominada Photo Tag Suggest amplamente utilizada a partir do final de 2010.

E precisamente a partir desta convergência entre a realidade aumentada e identificação facial, juntamente com os arquivos de consumo digitais que se gera uma preocupação relevante à efetividade da privacidade do consumidor-cidadão.

Estima-se, de acordo com alguns autores, mais de 1,35 bilhões de pessoas utilizam ativamente o Facebook, com mais de 250 bilhões de fotos postadas, inclusive como a identificação da foto do perfil, visto que a rede social utiliza-se de uma política de "identidade real", para que a pessoa mantenha a veracidade de suas informações pessoais (inclusive a foto) sob risco de exclusão da conta ${ }^{19}$. Portanto, todas podem ser identificadas a partir de um sistema desenvolvido de reconhecimento facial e que acesse estes dados.

Vale destacar que este tema representa uma discussão que tende a se tornar ainda mais relevantes em razão da capacidade de processamento das novas tecnologias, bem como a quantidade de imagens que são postadas online. Por exemplo, como mencionado por Acquisti et al., em 2000 aproximadamente 100 bilhões de fotos foram tiradas ao redor do mundo, ao tempo em que em 2012, apenas no Facebook foram postadas 300 milhões de fotos por dia, com a marcação de 100 milhões de pessoas diariamente ${ }^{20}$, alimentando um arquivo de dados com a imagem do rosto de bilhões de pessoas, relacionando-a ainda aos dados pessoais,

\footnotetext{
${ }^{18}$ WELINDER, Yana, op. cit., 2012. p. 171.

${ }^{19}$ ACQUISTI, Alessandro; GROSS, Ralph; STUTZMAN, Fred, op. cit., 2014. p. 1.

${ }^{20} \mathrm{Idem}$
} 
locais, datas e horas de cada foto tirada ${ }^{21}$.

Soma-se, ainda, às 75 milhões de fotografias em posse do departamento do Estado norte-americano, ou ainda à base de dados do Flickr com 3,4 bilhões de imagens e os 7,2 bilhões em posse da Photobucket ${ }^{22}$. Inaugura-se, deste modo, uma preocupação jurídica envolvendo a referida realidade aumentada e identificação facial, visto que apesar de realizar esta interação entre os dados virtuais e o contexto físico a que se propõe, pode materializar ofensas aos direitos dos cidadãos e consumidores.

Destaca-se que este risco à privacidade não se atém mais às relações de consumo propriamente ditas, ou mesmo às práticas comerciais envolvendo determinado produto ou serviço, porque qualquer pessoa que transita em local público pode ser alvo de investigações instantâneas e completas ${ }^{23}$.

Como se discorreu anteriormente, por se tratar de inovação tecnológica, ainda não se consegue esgotar todas as repercussões que seu desenvolvimento pode desencadear, existindo um esboço do seu potencial benéfico ou maléfico, como a identificação de terroristas em uma multidão, possibilidade de fraudes e estelionato, entre outros.

Diante destas possibilidades, em 2012, o senador norte-americano Al Franken entrou em contato com o próprio Facebook e o FBI para que fosse revista a utilização desta tecnologia de reconhecimento facial ${ }^{24}$, evidenciando, portanto, a necessidade de atuação estatal diante desta realidade aumentada e interação com o meio virtual, inclusive com uma releitura dos direitos fundamentais e da personalidade dos cidadãos, harmonizando-as ainda com os benefícios e os riscos do desenvolvimento tecnológico.

\subsection{A INTERNET DAS COISAS, DISPOSITIVOS MÓVEIS E SEUS APLICATIVOS E TECNOLOGIA WEARABLE}

As tecnologias descritas anteriormente contribuíram para o aprimoramento da revolução tecnológica que vem alterando significativamente o modo de interação interpessoal e com o próprio meio no qual se insere o ser humano e, ainda,

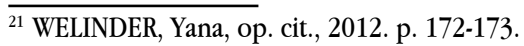

${ }^{22}$ LEVASHOV, Kirill, op. cit., 2013. p. 170.

${ }^{23}$ Conforme alerta Levashov: A concern that unavoidably arises from the use of facial recognition technology is that identifying information can be collected and stored en masse without the need for any physical restraint or contact; as a result, a person is vulnerable to having identifying information captured and stored by the government or a private company (or even an individual) just by appearing in public. Ibidem, 2013, p. 172.

${ }^{24}$ Ibidem, 2013. p. 189.
} 
readequando diversos objetos e atividades cotidianas que não se pode esquecer o seu potencial invasivo diante de dados sensíveis e que tal potencial merece ser reanalisado também sob uma tutela da privacidade e demais direitos consumeristas.

Assim, foi possível o desenvolvimento da "Internet das coisas" (do inglês, Internet of things), por meio da qual a rede mundial não se limita mais ao uso dos computadores, estendendo o seu uso para objetos e sistemas inteiros como carros, geladeiras, balanças, relógios, joias, óculos, entre outros. Muitas vezes relacionado ao termo "smart", que além de qualificar os objetos, smartphone, TV smart, geladeira smart, passam a caracterizar também sistemas integrados de objetos e pessoas, como "smart buildings, smart appliances, smart bealth, smart mobility, smart cities", entre outros ${ }^{25}$.

De acordo com Thierer, trata-se de uma geração de "objetos encantados" que passam a integrar uma conexão e exercem sua função de maneira sensitiva, automatizada e integrada, materializando esta denominada Internet das coisas ${ }^{26}$.

Pesquisas apontam que até 2020, 37 bilhões de objetos inteligentes estarão conectados e transmitindo e colhendo informações ${ }^{27}$, o que movimentaria ainda uma economia estimada em US\$ 14,4 trilhões entre o período de 2013 e $2022^{28}$.

Em outras palavras, significa dizer que os arquivos de consumo não se limitam mais apenas ao crédito e dados pessoais, estendendo-se também a comportamentos e costumes cotidianos que passam a ser registrados e muitas vezes compartilhados por esta rede integrada. Esta plena e ininterrupta conectividade dos cidadãos consumidores pode ser exemplificada também pelo uso dos dispositivos móveis que os acompanham diariamente, como os celulares, tablets e agora dispositivos usados também como vestimenta.

Estatisticamente, tem-se que $90 \%$ dos norte-americanos possuem um celular, quase $60 \%$ smartphone e $42 \%$ um tablet $^{29}$, e no Brasil alguns dados apontam que o número de celulares já superou a quantidade de habitantes ${ }^{30}$, cujas funcionalidades

\footnotetext{
25 THIERER, Adam D. The internet of things and wearable technology: addressing privacy and security concerns without derailing innovation. Richmond Journal of Law \& Technology, v. 21, n. 2, 2015, p. 10.

${ }^{26}$ Segundo o autor esta chamada Internet das Coisas, ou conectividade e comunicações máquina a máquina promete inaugurar "uma terceira revolução da computação" e trazer mudanças profundas que irão rivalizar com a primeira onda de inovação da Internet". Ibidem, 2015, p. 6-7.

${ }^{27}$ Ibidem., 2015, p. 12.

${ }^{28}$ Ibidem, 2015, p. 15.

${ }^{29}$ ARNOLD, Michael; KIKER, Dennis R. The Big Data Collection Problem of Little Mobile Devices. Richmond Journal of Law \& Technology, v. 21, n. 3, 2015, p. 2.

${ }^{30}$ Disponível em: <http://www.tecmundo.com.br/celular/26912-brasil-ja-tem-mais-celulares-ativos-do-que -habitantes.htm>. Acesso em: 30 jul. 2015.
} 
incluem a própria comunicação telefônica, mensagens de texto, fotografias, gravação de voz e vídeo, anotações pessoais e armazenagem de arquivos, utilização de mapas, rotas e GPS.

Por óbvio que não se pode desconsiderar as inúmeras vantagens provenientes deste avanço tecnológico e seu uso massificado, contudo, alguns pontos sensíveis quanto à privacidade devem ser devidamente estudados para que permaneçam sob a tutela prevista constitucionalmente.

$\mathrm{Na}$ Harvard Journal of Law and Technology, Cocker explica que mesmo diante da variação tecnológica, os dispositivos móveis funcionam com uma transmissão em duas vias com uma estrutura fixa e por meio de tecnologia sem fio, registrando-se todas estas atividades exercidas por meio destes dispositivos, de modo que podem gerar dados que podem ser usados para localização e investigação de tempo e data, sendo necessária uma regulamentação que entenda estes riscos, visto que whatever the doctrine or statutory scheme, it is clear that failing to place reasonable, meaning ful limits on the use of location data undermines public trust in a technology that increasingly enables communication, free association, and self-expression ${ }^{31}$.

Além desta possível localização, imediata ou até mesmo histórica, dos cidadãos por meio das tecnologias de triangulação de sinais para transmissão de dados, ou mesmo pelo GPS do aparelho, que inclusive tem despertado polêmica quanto à privacidade no trabalho ${ }^{32}$, há ainda a análise de informações decorrentes do uso de aplicativos neles instalados.

Em estudo empírico realizado por Liccardi et al., no qual se analisou a política de privacidade de $\mathbf{5 2 8 . 4 3 3}$ aplicativos, representando $88 \%$ da playstore para dispositivos móveis com sistema Android, constatou-se que apenas 6,6\% destes

\footnotetext{
${ }^{31}$ Tradução livre: "qualquer que seja a doutrina ou regime legal, é claro que não colocar limites razoáveis e significativos sobre a utilização de dados de localização mina a confiança pública em uma tecnologia que permite a comunicação cada vez mais, a associação livre e auto-expressão". CROCKER, Andrew. Trackers that make phone calls: considering first amendment protection for location data. Harvard Journal of Law and Technology, v. 26, n. 2, 2013, p. 625.

${ }^{32}$ The data on any given device may originate with the user, an employer, or another third party, or be collected through automatic means (for example, through data logging, geolocation tracking, or built-in motion detectors). 12 The Supreme Court's opinion in Riley v. California highlighted the "element of pervasiveness that characterizes cell phones" as well as the quantity and quality of data that they contain in its discussion of just how integral today's smartphones are to modern life, and the various purposes for which they are used (MCLELLAN, Melinda L.; SHERER, James A.; FEDELES, Emily R. Wherever You Go, There You Are (With Your Mobile Device): Privacy Risks and Legal Complexities Associated with International "Bring Your Own Device" Programs. Richmond Journal of Law \& Technology, v. 21, n. 03, 2015, p. 3-4).
} 
aplicativos possuem uma política de privacidade disponível na playstore ${ }^{33}$, ou seja antes de realizar a instalação deste aplicativo. Isto representa consequentemente que mais de 93\% dos aplicativos não explicam claramente como usarão os dados pessoais disponíveis no aparelho e coletados no decorrer de seu uso ${ }^{34}$.

$\mathrm{E}$, curiosamente, mesmo em aplicativos de uma mesma categoria, aplicativos de wallpaper, percebe-se que os aplicativos grátis requerem uma quantidade maior de dados e de natureza mais sensível do que os aplicativos que exercem esta mesma função e são pagos pelo usuário, podendo-se deduzir que parte do lucro destas empresas que desenvolvem aplicativos grátis decorrem tanto da publicidade neles presentes quanto da exploração deste vasto conjunto de informações sobre seus usuários ${ }^{35}$.

Fato que tem se agravado em razão da popularização da wearable technology, ou dispositivos que passam a ser incorporados na vestimenta dos consumidores. Novamente se ressalva que em outra proporção esta ideia já tem sido desenvolvida há décadas, como o relógio com calculadora, aparelhos auditivos, entre outros, mas que devem ser reanalisados em razão de seu modo de processamento e transmissão de dados.

Trata-se de segmento que cresceu $700 \%$ apenas do primeiro semestre até o final de 2013, e tendem a revolucionar o modo como se interage com o meio, se pratica atividade física ou mesmo a medicina, visto que As they grow more sophisticated, wearable bealth devices will help users track, and even diagnose various conditions, and potentially advise a course of action or, more simply, remind users to take medications or contact medical professionals as necessary ${ }^{36}$.

Estima-se, ainda, que referida tecnologia tem uma capacidade evolutiva já em transação, com a comercialização e popularização de Implantables, Ingestibles e Biobacking ${ }^{37}$, podendo representar alterações paradigmáticas no que tange à tecnologia e os limites humanos do corpo, até mesmo da vida, devendo ser igualmente precedida por uma compreensão desta permuta entre vida cotidiana e

\footnotetext{
${ }^{33}$ LICCARDI, Ilaria; PATO, Joseph; WEITZNER, Daniel J. Improving User Choice Through Better Mobile Apps Transparency and Permissions Analysis. Journal of Privacy and Confidentiality, v. 5, n. 2, Article 1. 2014. p. 9.

${ }^{34}$ Ibidem, 2014. p. 10.

${ }^{35}$ Ibidem, 2014. p. 19.

${ }^{36}$ Tradução livre: À medida que crescem mais sofisticados, dispositivos de saúde vestíveis irão ajudar os usuários a controlar, e até mesmo diagnosticar várias condições, e, potencialmente, aconselhar um curso de ação ou, mais simplesmente, lembrar os usuários a tomar medicamentos ou entrar em contato com profissionais da área médica, se necessário. THIERER, Adam D. op. cit., 2015, p. 19-20.

${ }^{37}$ Ibidem, 2015, p. 31.
} 
tecnologia, principalmente no que se refere à privacidade dos cidadãos.

\section{RELEITURA DA PRIVACIDADE}

Diante deste potencial transformativo decorrente das inovações tecnológicas, há uma constante ameaça à privacidade dos cidadãos-consumidores, sendo necessária, todavia, a efetiva compreensão deste conceito de privacidade. Deve-se ressalvar que diversos são os valores constitucionais envolvidos nesta discussão, visto que não se pode censurar a evolução tecnológica ou mesmo limitar injustificadamente a livre iniciativa, posto que em conjunto com a justiça social e defesa dos consumidores constitui princípio da ordem econômica.

Ao tratar do desenvolvimento tecnológico em uma sociedade de risco, Efing, Misugi e Bauer já discorreram que o desenvolvimento técnico e científico pode ser, em realidade, instrumento para a superação da emergência socioambiental, devendo ser harmonizado com os demais direitos fundamentais, como o direito ao meio ambiente ecologicamente equilibrado e a proteção dos direitos do consumidor e da personalidade ${ }^{38}$.

Ainda assim, alguns autores mencionados anteriormente, como Levashov, defendem que diante da utilização de informações biométricas, tem-se uma situação que exige uma regulamentação direta para que se previna o risco que se apresenta ${ }^{39}$.

Enaltece-se, em contrapartida, a construção teórica desenvolvida por Thierer (2015, p. 39-40), na qual se pondera exatamente os valores e riscos envolvendo uma política pública de controle tecnológico, apresentando duas teorias: permissionless innovation e the precautionary principle ${ }^{40}$. Entende-se por permissionless innovation o entendimento de que se deve realizar a experimentação das novas tecnologias e atividades mercadológicas sem a necessidade de autorização prévia, a menos que sua aplicação prática demonstre-se divergente do ordenamento jurídico vigente. No que se refere a the precautionary principle mencionado pelo autor, inverte-se esta lógica, exigindo-se dos desenvolvedores e beneficiários de determinada tecnologia a demonstração de sua segurança e adequação, defendendo, ainda, que com o desenvolvimento destas novas tecnologias urge-se a efetivação do direito à

\footnotetext{
${ }^{38}$ EFING, Antônio Carlos; MISUGI, Guilherme; BAUER, Fernanda Mara Gibran. O consumo consciente e o enfrentamento do risco do Desenvolvimento Tecnológico. In: Antonio Herman Benjamin; José Rubens Morato Leite. (Org.). Congresso Brasileiro de Direito Ambiental: ambiente, sociedade e consumo sustentável. São Paulo: Instituto O Direito por um Planeta Verde, 2015, v. 1, p. 78-95; p. 88-89.

${ }^{39}$ LEVASHOV, Kirill. op. cit., 2013, p. 193.

${ }^{40}$ THIERER, Adam D. op. cit., 2015, p. 53-54.
} 
informação mais eficiente e clara, perpassando ainda por uma resiliência por parte da sociedade, como exemplificado anteriormente no caso da máquina fotográfica, e uma atuação solidária dos usuários destas tecnologias. Em suma, Thierer aponta pela necessidade de um controle democrático:

The better alternative to top-down regulation is to deal with concerns creatively as they develop, using a combination of educational efforts, technological empowerment tools, social norms, public and watchdog pressure, industry best practices and self-regulation, transparency, and targeted enforcement of existing legal standards (especially torts), as needed. This bottom-up and layered approach to dealing with problems will not preemptively suffocate technological experimentation and innovation in these spaces ${ }^{41}$.

Por fim, o autor ainda ressalta que uma regulamentação precisa, seja preventiva ou na aferição de responsabilidade, é dificultada por uma imprecisão no conceito de privacidade, uma vez que representa valor subjetivo e de difícil compreensão.

Nacionalmente, por exemplo, curiosamente não se encontra o vocábulo privacidade no texto constitucional, no Código Civil de 2002, nem mesmo no Código de Defesa do Consumidor, sendo elevado, ainda assim, a direito fundamental posto que "são invioláveis a intimidade, a vida privada, a honra e a imagem das pessoas, assegurado o direito a indenização pelo dano material ou moral decorrente de sua violação". Existindo tutela similar no CDC, visto que tutela a integridade física e moral dos consumidores, sendo a privacidade valor essencial à personalidade destes. Já a Lei n ${ }^{0}$ 12.965/2014, denominada de Marco Civil da Internet, passou a se utilizar o termo privacidade, como princípio do uso da Internet no Brasil e condição para o pleno exercício do direito de acesso à Internet, figurando também na seção sobre "Proteção aos Registros, aos Dados Pessoais e às Comunicações Privadas", de modo que se assimila que a discussão da privacidade dos consumidores também pode convergir com a tutela sobre a criação e utilização dos arquivos de consumo, conforme já antecipava a discussão trazida por Efing $^{42}$, e complementada por Barbosa:

Trata-se, aqui, das informações armazenadas em bancos de dados digitais, cuja proliferação abusiva tem sido um dos maiores problemas

${ }_{41}^{41}$ Tradução livre: A melhor alternativa à regulamentação top-down é lidar com preocupações de forma criativa à medida em que elas se desenvolvem, usando uma combinação de esforços educacionais, ferramentas de capacitação tecnológica, normas sociais, pressão da opinião pública e de vigilância, melhores práticas da indústria e auto-regulação, transparência e cumprimento alvo normas jurídicas (especialmente aos factos) da existente, conforme necessário. Este bottom-up e abordagem em camadas para lidar com problemas não sufoca preventivamente experimentação tecnológica e inovação nestes espaços. THIERER, Adam D. op. cit., 2015, p. 3-4.

${ }^{42}$ EFING, Antônio Carlos. Banco de dados e cadastro de consumidores. São Paulo: Revista dos Tribunais, 2002. 
enfrentados pelos consumidores na atualidade, e que viola o que se tem convencionado chamar de 'direito à autodeterminação informacional' (ou 'liberdade informática', como na Alemanha, ou ainda, 'proteção de dados pessoais', como na Itália), entendido como 'o direito do cidadão de tomar conhecimento sobre o arquivamento e uso das informações suas por terceiros, bem como de controlá-los e mesmo impedi-los. A proliferação será considerada abusiva ou não tomando-se em conta dois aspectos: o tipo de dado divulgado e a forma de sua divulgação ${ }^{43}$.

Ainda assim, permanece uma justificada nebulosidade acerca do conceito e limites da privacidade diante de sua amplitude e dinamicidade, que pode ser considerado conexo ao da própria vida, figurando como reflexo ou manifestação deste, conforme define Silva:

De fato, a terminologia não é precisa. Por isso, preferimos usar a expressão direito à privacidade, num sentido genérico e amplo, de modo a abarcar todas essas manifestações da esfera íntima, privada e da personalidade, que o texto constitucional em exame consagrou. Toma-se, pois, a privacidade como 'o conjunto de informação acerca do indivíduo que ele poderia decidir manter sob o seu exclusivo controle, ou comunicar, decidindo a quem, quando, onde e em que condições, sem a isso poder ser legalmente sujeito'. A esfera da inviolabilidade, assim, é ampla, 'abrange o modo de vida doméstico, nas relações familiares e afetivas em geral, fatos, hábitos, local, nome, imagem, pensamentos, segredos, e, bem assim, as origens e planos futuros do indivíduo' ${ }^{44}$.

O autor aprofunda ainda aproximando-a ao denominado Right to be alone, o direito de toda pessoa tomar sozinha as decisões na esfera da sua vida privada, similar ao conceito complementado por Efing:

Este direito de ser deixado só, em paz, apesar de extremamente privatista, vem a expressar o verdadeiro condão da participação política do indivíduo enquanto cidadão, quando pretende garantir uma esfera privada indisponível e inatingível, senão pelos interesses de seu único titular, em consonância com os interesses sociais daí emergentes, hodiernamente representando verdadeira 'liberdade democrática' ${ }^{\prime 5}$.

Ou seja, trata-se de um direito fundamental que se torna imprescindível à vida digna e formação da própria personalidade do cidadão, sendo ela essencial também para o pleno exercício da cidadania e demais liberdades, até mesmo como

\footnotetext{
${ }^{43}$ BARBOSA, Fernanda Nunes. Informação e consumo: a proteção da privacidade do consumidor no mercado contemporâneo da oferta. Revista de Direito do Consumidor, São Paulo, v. 22, n. 88, p. 103-143, jul. 2013. p. 151.

${ }^{44}$ SILVA, José Afonso da. Curso de direito constitucional positivo. 33. ed. São Paulo: Malheiros, 2010, p. 206.

${ }^{45}$ EFING, Antônio Carlos. op. cit., 2002, p. 51.
} 
consumidor, integrando também conceito amplo que engloba o direito à intimidade, à vida privada e à imagem.

Ao se retomar o trabalho de Warren e Brandeis (1890), na avaliação dos autores, a solidão e a privacidade tornaram-se mais essencial para o indivíduo, porém com cada vez mais invenções agindo sobre a vida privada, o indivíduo poderia submeter-se à dor e angústia mental, sendo estas muito maiores do que as que poderiam ser causadas por mera lesão corporal ${ }^{46}$. Por isso, os autores defendem que se a privacidade for reconhecida como um direito à proteção legal, a interposição dos tribunais não poderia vir a depender da natureza particular das lesões resultantes ${ }^{47}$. É interessante notar que, à época, os autores já mencionavam a necessidade de existência de cláusula contratual, implicando em relação de confiança ${ }^{48}$. Finalmente, os autores afirmam que a "lei comum sempre reconheceu a casa de um homem como o seu castelo, inexpugnável, muitas vezes, até mesmo para seus próprios oficiais envolvidos na execução do seu comando" e perguntam se "Porventura os tribunais não estariam a fechar a porta da frente com a autoridade constituída e a abrir a porta dos fundos para a ampla curiosidade ou lasciva?”49 . A pergunta é provocadora e atual.

E mesmo com a elucidação doutrinária, principalmente diante da globalização e mitigação das barreiras geográficas e culturais características da sociedade da informação, pode-se conceber uma fragilização da proteção da privacidade, principalmente nas relações virtuais e de consumo, que merecem maior atenção dos operadores do Direito e dos formadores de políticas públicas.

Esta dificuldade de conceituação, e mesmo efetivação, decorre de uma noção superficial, ou mesmo equivocada, de que a privacidade é um valor absoluto, mundial, e estático que se materializa diante de "argumentos intuitivos" ${ }^{50}$, gerando, consequentemente, uma tutela igualmente intuitiva e que desconsidera a amplitude do tema e ao mesmo tempo favorece a contradição entre os conceitos multiculturais da privacidade, os quais se tornam cada vez mais relevantes diante do estreitamento das relações internacionais.

A releitura a que se propõe o presente estudo, portanto, não pretende esgotar a discussão ou mesmo solidificar um conceito atualizado de privacidade, uma vez que se defende exatamente esta dinamicidade da privacidade, que possui

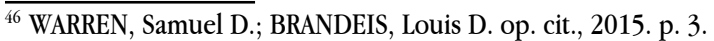

${ }^{47}$ Ibidem, 2015. p. 7.

${ }^{48}$ Ibidem, 2015. p. 9.

${ }^{49}$ Ibidem, 2015. p. 15.

${ }^{50}$ WHITMAN, James Q. The Two Western Cultures of Privacy: Dignity versus Liberty. Yale Law Journal, v. 113, April 2004. p. 1154.
} 
influências multiculturais e cuja tutela deve dar-se por meio de uma incidência também dinâmica do Direito e do solidarismo de seus agentes.

Para se exemplificar como a privacidade pode ser interpretada de maneira distinta, por vezes até contraditória, Whitman (2004) realizou ampla pesquisa acerca deste direito fundamental a partir de duas culturas ocidentais distintas, a privacidade nos Estados Unidos da América, e sua noção nos países europeus, principalmente França e Alemanha. O que se concluiu foi que a própria concepção da privacidade tem origens e fundamentos distintos, pois enquanto a privacidade norte-americana deriva de uma noção de liberdade, na Europa tal direito tem como fundamento a dignidade do sujeito ${ }^{51}$.

Torna-se essencial esclarecer que esta distinção não se trata de contraste absoluto, representando apenas uma concepção de privacidade que, ao se utilizar de metáforas, podem transitar sob órbitas gravitacionais distintas, mas por vezes permeiam um espaço coincidente.

Alguns fatos ilustram esta distinção entre o que seria considerado um risco à privacidade, como a maneira que se é culturalmente tratada a nudez, os dados dos consumidores $^{52}$, a escolha de nome dos filhos ou mesmo a divulgação de renda dos trabalhadores. Por exemplo, In France and Germany, according to a recent study, telephones are tapped at ten to thirty times the rate they are tapped in the United States - and in the Netherlands and Italy, at 130 to 150 times the rate ${ }^{53}$.

Ainda que o mencionado estudo apresente as justificativas históricas e demais fatos interessantes para a compreensão desta multidimensão da privacidade, deve-se enaltecer que At least as far as the law goes, we do not seem to possess general "buman" intuitions about the "borror" of privacy violations ${ }^{54}$.

Outro ponto relevante a ser analisado refere-se também à própria ideia

\footnotetext{
${ }^{51}$ Como descreve Whitman: Continental privacy protections are, at their core, a form of protection of a right to respect and personal dignity. The core continental privacy rights are rights to one's image, name, and reputation, and what Germans call the right to informational self-determination - the right to control the sorts of information disclosed about oneself. These are closely linked forms of the same basic right: They are all rights to control your public image - rights to guarantee that people see you the way you want to be seen. They are, as it were, rights to be shielded against unwanted public exposure - to be spared embarrassment or humiliation. The prime enemy of our privacy, according to this continental conception, is the media, which always threatens to broadcast unsavory information about us in ways that endanger our public dignity. [...] By contrast, America, in this as in so many things, is much more oriented toward values of liberty, and especially liberty against the state. At its conceptual core, the American right to privacy still takes much the form that it took in the eighteenth century: It is the right to freedom from intrusions by the state, especially in one's own home. (WHITMAN, James Q. op. cit., 2004. p. 1162).

52 Ibidem, 2004. p. 1192.

53 Tradução livre: "Na França e na Alemanha, de acordo com um estudo recente, os telefones são grampeados em dez a trinta vezes a taxa que nos Estados Unidos, e na Holanda e na Itália, em 130 a 150 vezes esta taxa". Ibidem, 2004. p. 1159.

${ }^{54}$ Tradução livre: "Pelo menos na medida da lei, nós não parecemos possuir intuições 'humanas' e gerais sobre o 'horror' de violações de privacidade". Ibidem, 2004. p. 1160.
} 
popular de fragilização voluntária da privacidade pelos cidadãos, mormente os usuários das redes sociais que buscam a exposição de suas vidas, existindo uma evolução (ou retrocesso) temporal na sua valorização subjetiva.

Ocorre que esta premissa de que os usuários de redes sociais cada vez menos preocupam-se com sua privacidade, fragilizando o valor jurídico desta, demonstra-se falsa, conforme se comprovou empiricamente.

Em estudo realizado por Stutzman, Gross e Acquisti, pôde-se concluir que desde o início da utilização do Facebook, em 2005, até 2011, foram evidenciadas três tendências relevantes para a compreensão desta valoração da privacidade: a) Com o tempo, os usuários aumentaram o privacy-seeking behavior, diminuindo a quantidade de informações disponíveis publicamente; b) Mudanças na política de privacidade da rede social conseguiram amenizar ou inverter esta tendência no comportamento dos seus usuários; c) Ao contrário das informações públicas, houve um acréscimo na comunicação e troca de informações privadas entre os usuários, aumentando consequentemente a quantidade de informações armazenadas pela rede social e os denominados silent listeners ${ }^{55}$.

Deduz-se, desta maneira, que ao se inserir a presente discussão, interpretação holística e dinâmica da privacidade, em uma sociedade de consumo globalizada, caracterizada também como sociedade da informação, na qual as evoluções tecnológicas gradualmente processam e armazenam mais dados acerca dos cidadãos consumidores, é essencial que se tenham mecanismos jurídicos igualmente atuais para sua tutela.

A partir destas informações, gera-se uma noção de expectativa social que começa a balizar o conceito de privacidade, considerando tanto a natureza dos dados, públicos, pessoais, sensíveis ou não, bem como a forma de transmissão e destinatários.

Teoria que encontra similar repercussão no ordenamento jurídico brasileiro, visto que Barbosa defende que a privacidade "deixa de se estruturar no eixo pessoainformação-segredo e passa a se reestruturar no eixo pessoa-informação-controle" ${ }^{n 6}$.

Representa, inclusive, um dos temas de atualização do CDC, mediante a PLS 281/2012, cuja justificativa aponta que visa "a fortalecer a sua confiança e assegurar tutela efetiva, preservar a segurança nas transações, a proteção da autodeterminação e da privacidade dos dados pessoais; as normas aplicam-se às atividades desenvolvidas pelos fornecedores de produtos ou serviços por meio

\footnotetext{
${ }^{55}$ STUTZMAN, Fred; GROSS, Ralph; ACQUISTI, Alessandro. Silent Listeners: The Evolution of Privacy and Disclosure on Facebook, Journal of Privacy and Confidentiality, v. 4, n. 2, Article 2. 2013.

${ }^{56}$ BARBOSA, Fernanda Nunes, op. cit.,. 2013, p. 150.
} 
eletrônico ou similar", e pretende incluir dentre os direitos básicos do consumidor, no art. $6^{\circ}$, "XI - a autodeterminação, a privacidade e a segurança das informações e dados pessoais prestados ou coletados, por qualquer meio, inclusive o eletrônico" e "XII - a liberdade de escolha, em especial frente a novas tecnologias e redes de dados, sendo vedada qualquer forma de discriminação e assédio de consumo".

Conduz-se, deste modo, a uma necessária releitura do direito à privacidade, visto que, conforme exposto anteriormente, não pode ser considerado como valor absoluto e estático, devendo ser constantemente reinterpretado, principalmente em razão das novas tecnologias, considerando as influências multiculturais que invariavelmente permearão esta discussão.

Para tal objetivo, reitera-se a natureza principiológica da tutela consumerista, bem como a eficácia horizontal dos direitos fundamentais, para que a privacidade e o desenvolvimento tecnológico desenvolvam-se em harmonia, sendo tutelados principalmente por um solidarismo entre os desenvolvedores das novas tecnologias e seus usuários e fornecedores que delas se utilizam.

\section{CONSIDERAÇÕES FINAIS}

A configuração da atual sociedade de consumo como uma sociedade da informação acarreta diversas consequências para os cidadãos consumidores, visto que além da agilidade com que são processadas as informações, estas passam a possuir valoração econômica, especialmente aquelas de carácter pessoal e/ou sensíveis, evidenciando características relacionadas à sua intimidade e privacidade.

E o direito como ciência social deve acompanhar referida alteração paradigmática, de modo a dar efetividade às tutelas constitucionais e consumeristas previstas ao cidadão consumidor e, portanto, carecendo de uma constante reinterpretação de seus valores para sua efetiva tutela e consequentemente seu respeito. E tendo em vista que a tutela já se encontra prevista no ordenamento jurídico brasileiro e a dificuldade da atualização legislativa no ritmo da evolução tecnológica, enaltece-se a hermenêutica principiológica destes direitos fundamentais para que permaneçam sempre eficazes.

Neste cenário, as novas tecnologias que têm sido desenvolvidas e alterarão todo o mercado de consumo merecem especial atenção no que se refere a este desenvolvimento tecnológico sustentável sob o ponto de vista social, cultural e jurídico.

Como exemplo, foram apontados a popularização da realidade aumentada 
e o reconhecimento facial, os dispositivos móveis e suas particularidades, entre outros, exemplificando que a inovação tecnológica é constante e acelerada a cada nova geração.

Logo, as preocupações tecnológicas e informacionais, outrora limitadas a um ambiente estritamente computadorizado, passam a se estender a qualquer ambiente social, visto que com estas novas tecnologias - principalmente a realidade aumentada e a Internet das coisas - enfraquecem-se os aspectos de segurança das informações, a saber: confidencialidade, integridade, disponibilidade, autenticidade e não repúdio; sendo que tais aspectos são primordiais à tutela jurídica do direito à privacidade.

Razão pela qual, podendo-se analisar até a personalidade do cidadão consumidor no ambiente digital (seus interesses, gostos e preferências), tem-se em constante conflito os direitos fundamentais e inerentes à personalidade frente às novas tecnologias e capacidade de processamento e armazenamento de dados, incorrendo em um grave risco principalmente à privacidade daqueles.

Como a pretensão do presente estudo não era esgotar o tema, apresentaramse diversas dimensões multiculturais relacionadas à privacidade, evidenciando que se trata de um valor dinâmico e multidimensional, alterado inclusive pela própria utilização das tecnologias de informação e comunicação (TIC).

Finalmente, entende-se que a tutela da privacidade diante das novas tecnologias deve ser igualmente resiliente e que incida de maneira principiológica, propondo-se a aplicação da teoria da contextual integrity, para que se analise o contexto e natureza das informações em questão e, portanto, a tutela possa ser norteada, principalmente, pelo solidarismo entre os agentes envolvidos nas relações públicas e privadas.

\section{REFERÊNCIAS}

ACQUISTI, Alessandro; GROSS, Ralph; STUTZMAN, Fred. Face recognition and privacy in the age of augmented reality. Journal of Privacy and Confidentiality, v. 6, n. 2, 2014.

ARNOLD, Michael; KIKER, Dennis R. The big data collection problem of little mobile devices. Richmond Journal of Law \& Technology, v. 21, n. 03, 2015. 
BARBOSA, Fernanda Nunes. Informação e consumo: a proteção da privacidade do consumidor no mercado contemporâneo da oferta. Revista de Direito do Consumidor, São Paulo, v. 22, n. 88, p. 103-143, jul. 2013.

BARRETO JUNIOR, Irineu Francisco. Abordagens recentes da pesquisa jurídica na Sociedade da Informação. In: PAESANI, Liliana Minardi. (Coord.). O direito na sociedade de informação II. São Paulo: Atlas, 2009.

BENJAMIN, Antônio Herman et al. Código brasileiro de defesa do consumidor: comentado pelos autores do anteprojeto. 9. ed. Rio de Janeiro: Forense Universitária, 2007.

BENJAMIN, Antonio Herman V. et al. Código Brasileiro de defesa do consumidor: comentado pelos autores do anteprojeto. 10. ed. revista, atualizada e reformulada. Rio de Janeiro: Forense, 2011. Vol. I. p. 260.

BRASIL. Congresso Nacional. Constituição da República Federativa do Brasil, 1988. Disponível em: <http://www.planalto.gov.br/ccivil_03/constituicao/constituicao. htm >. Acesso em: 15 ago. 2015.

BRASIL. Lei $\mathrm{n}^{\mathrm{o}}$ 10.406, de 10 de janeiro de 2002. Disponível em: < http://www. planalto.gov.br/ccivil_03/leis/2002/L10406.htm > . Acesso em: 15 ago. 2015.

BRASIL. Lei $\mathrm{n}^{\mathrm{O}} 12.965$, de 23 de abril de 2014. Disponível em: < http://www.planalto. gov.br/ccivil_03/_ato2011-2014/2014/lei/112965.htm >. Acesso em: 15 ago. 2015.

BRASIL. Planalto. Lei no 8.078, de 11 de setembro de 1990. Disponível em: < http:// www.planalto.gov.br/ccivil_03/Leis/L8078.htm > . Acesso em: 15 ago. 2015.

BRASIL. Senado. Projeto de Lei do Senado, $\mathrm{n}^{\circ} 281$ de 2012. Disponível em: < http:// www.senado.gov.br/atividade/materia/detalhes.asp?p_cod_mate $=106768>$. Acesso em: 15 ago. 2015.

BRASIL. Senado. Projeto de Lei do Senado, $\mathrm{n}^{\circ} 330$ de 2013. Disponível em: < http:// www.senado.gov.br/atividade/materia/detalhes.asp?p_cod_mate $=113947>$. Acesso em: 15 ago. 2015. 
CASTELLS, Manuel. A sociedade em rede. 7. ed. totalmente rev. e ampl. Tradução de Roneide Venancio Majer com a colaboração de Klauss Brandini Gerhardt. São Paulo: Paz e Terra, 2003. Vol I.

CROCKER, Andrew. Trackers that make phone calls: considering first amendment protection for location data. Harvard Journal of Law and Technology, v. 26, n. 2, 2013.

EFING, Antônio Carlos. Banco de dados e cadastro de consumidores. São Paulo: Revista dos Tribunais, 2002.

EFING, Antônio Carlos; MISUGI, Guilherme; BAUER, Fernanda Mara Gibran. O consumo consciente e o enfrentamento do risco do Desenvolvimento Tecnológico. In: Antonio Herman Benjamin; José Rubens Morato Leite. (Org.). Congresso Brasileiro de Direito Ambiental: Ambiente, sociedade e consumo sustentável. São Paulo: Instituto O Direito por um Planeta Verde, 2015, v. 1, p. 78-95.

GIBRAN, Fernanda Mara; EFING, Antônio Carlos. Banco de Dados de Consumo como instrumento para o desenvolvimento da sociedade de informação. In: ENCONTRO NACIONAL DO CONPEDI, 19., 2010, Fortaleza. Anais... Fortaleza: Conpedi, 2010. p. 3886-3895.

GREENFIELD, Adam. Everyware: the dawning age of ubiquitous computing. AIGA: New Riders, 2006.

KURZWEIL, Ray. The singularity is near: when humans transcend biology. London: Penguin Books, 2005.

LEVASHOV, Kirill. The Rise of a New Type of Surveillance for Which the Law Wasn't Ready. Columbia Science and Technolog y Law Review, v. 15, p. 164, fall 2013.

LICCARDI, Ilaria; PATO, Joseph; WEITZNER, Daniel J. Improving User Choice Through Better Mobile Apps Transparency and Permissions Analysis. Journal of Privacy and Confidentiality, v. 5, n. 2, 2014.

LISBOA, Roberto Senise. O consumidor na sociedade da informação. Revista de Direito do Consumidor, n. 61, jan. 2007. 
LISBOA, Roberto Senise. Tecnologia, confiança e sociedade. Por um novo solidarismo. In: PAESANI, Liliana Minardi. (Coord.). $O$ direito na sociedade de informação II. São Paulo: Atlas, 2009.

MAIA, Maurilio Casas. Telemedicina, prontuário eletrônico e atualização do Código de Defesa do Consumidor: a tutela da hipervulnerabilidade eletrônica do paciente e de sua personalidade virtual. Revista de Direito do Consumidor, São Paulo, v. 22, n. 89, p. 303-319, set. 2013.

MARQUES, Cláudia Lima. A proteção dos consumidores em um mundo globalizado: Studium Generale sobre o consumidor como homo novus. Revista de Direito do Consumidor, São Paulo, v. 22, n. 85, p. 25-63, jan. 2013.

MCLELLAN, Melinda L.; SHERER, James A.; FEDELES, Emily R. Wherever You Go, There You Are (With Your Mobile Device): Privacy Risks and Legal Complexities Associated with International "Bring Your Own Device" Programs. Richmond Journal of Law \& Technology, v. 21, n. 03, 2015.

MIRAGEM, Bruno. Responsabilidade por danos na sociedade de informação e proteção do consumidor: desafios atuais da regulação jurídica da Internet. Revista de Direito do Consumidor, São Paulo, n. 70, p. 41-92, abr. 2009.

MISUGI, Guilherme; EFING, Antônio Carlos. O respeito aos direitos do cidadão consumidor como pressuposto ao desenvolvimento socioambiental. In: KNOERR, Viviane Coêlho de Séllos; MARTINS, Fernando Rodrigues; HAONAT, Angela Issa. (Org.). Direito do consumidor. Florianópolis: CONPEDI, 2014, v. 23, p. 392-412.

MOUTINHO, Ana Maria. Inteligência ambiente: contributo para a conceptualização de parede inteligente. 2010. Dissertação (Mestrado) - Universidade de Lisboa, Lisboa, 2010. Disponível em < http://repositorio.ul.pt/bitstream/10451/7277/2/ ULFBA_tes\%20392.pdf> Acesso em: 30 jul. 2015.

ORITO, Yohko; MURATA, Kiyoshi. Privacy Protection in Japan: Cultural Influence on the Universal Value. ETHICOMP 2005, Linköping, Sweden. p. 04. Disponível em: $<$ http://www.kisc.meiji.ac.jp/ ethicj/Privacy\%20protection\%20in\%20Japan.pdf>. Acesso em: 20 jul. 2015. 
PARCHEN, Charles Emmanuel; FREITAS, Cinthia Obladen de Almendra; EFING, Antônio Carlos. Computação em nuvem e aspectos jurídicos da segurança da informação. Revista Jurídica CESUMAR. Mestrado, v. 13, p. 397-417, 2013.

PASQUALE, Frank; RAGONE, Tara Adams. Protecting Health Privacy in an Era of Big Data Processing and Cloud Computing. Stanford Technology Law Review, v. 17, september, 2014.

PINHEIRO, Patrícia Peck. Direito Digital. 4. ed. rev., atual. e ampl. São Paulo: Saraiva, 2010.

RODRIGUES, Georgete Medleg; ANDRADE, Patrícia Simas; SIMÃO, João Batista. Sociedade da Informação no Brasil e em Portugal: um panorama dos livros verdes. Revista Ciência da Informação. v. 32, n. 3, set./dez. 2003. Disponível em: < http:// revista.ibict.br/index.php/ciinf/article/view/16> Acesso em: 30 jul. 2015.

SILVA, José Afonso da. Curso de direito constitucional positivo. 33. ed. São Paulo: Malheiros, 2010.

STUTZMAN, Fred; GROSS, Ralph; ACQUISTI, Alessandro. Silent listeners: the evolution of privacy and disclosure on Facebook. Journal of Privacy and Confidentiality, v. 4, n. 2, p. 7-41, 2013.

THIERER, Adam D. The internet of things and wearable technology: addressing privacy and security concerns without derailing innovation. Richmond Journal of Law \& Technology, v. 21, n. 2, 2015.

WARREN, Samuel D.; BRANDEIS, Louis D. The Right to Privacy. Harvard Law Review, v. 4, n. 5, 1890. Disponível em: < http://faculty.uml.edu/sgallagher/Brandeisprivacy. htm> Acesso em: 30 jul. 2015.

WEISER, Mark. Some computer science issues in ubiquitous computing. Communications of the ACM. Disponível em: <http://www.ubiq.com/hypertext/ weiser/UbiCACM.html>. Acesso em: 20 out. 2014.

WELINDER, Yana. A face tells more than a thousand posts: developing face recognition privacy in social networks. Harvard Journal of Law \& Technology, Boston, v. 26, 
n. 1, fall 2012.

WHITMAN, James Q. The two western cultures of privacy: dignity versus liberty. Yale Law Journal, v. 113, april 2004.

Recebido em: 31 de agosto de 2015 Aceito em: 28 de março de 2016 